\title{
The Role of Microalbuminuria in Arterial Endothelial Dysfunction in Hypertensive Patients With Carotid Plaques
}

\author{
Lin Yun, ${ }^{1,2}$ MD, Rui Xu, ${ }^{1}$ MD, Li Zhang, ${ }^{3}$ MD, Guohua LI, ${ }^{1}$ MD, Shuai HuAng, ${ }^{1}$ MD, \\ Yucai YAO, ${ }^{1} \mathrm{MD}$, and Jiamin LI, ${ }^{1} \mathrm{MD}$
}

\begin{abstract}
SUMMARY
The aim of this study was to evaluate the correlations between microalbuminuria (MAU) and endothelial function in hypertensive patients with carotid plaques (CP). A total of 71 hypertensive patients with CP (CP group) and 20 healthy people as normal controls (NC group) were enrolled in this study. The CP group was divided into an MAU group (MAU group, $n=33$ ) and a non-microalbuminuria group (NM group, $n=38$ ) according to their urinary albumin excretion rates (UAER). Endothelial function was assessed by flow-mediated dilation (FMD) in the brachial artery by ultrasonography, and nitroglycerin-mediated dilation (NMD) was used as a control test for FMD. Intima-media thickness (IMT) and biochemical parameters were evaluated. The $\triangle \mathrm{FMD} \%$ was significantly lower in patients with and without MAU in the CP group compared to the NC group. All patients with MAU had significantly lower $\triangle \mathrm{FMD} \%$ and $\triangle \mathrm{NMD} \%$ compared to the patients without MAU. $\triangle \mathrm{FMD} \%$ showed significant negative correlations with IMT, systolic blood pressure, glucose, total cholesterol, low density lipoprotein, high-sensitivity C-reactive protein, and log UAER. $\triangle$ NMD $\%$ demonstrated significant negative correlations with age, IMT, systolic blood pressure, glucose, and log UAER. Stepwise multiple linear regression analysis revealed that FMD was independently correlated with UAER and total cholesterol, while NMD was independently correlated with UAER and age. These results suggest that MAU might aggravate the arterial dysfunction and play a role in the arterial endothelial function in patients with hypertension and CP. Both endothelium-dependent and endothelium-independent vasodilatations were impaired in hypertensive patients with CP. (Int Heart J 2014; 55: 153-159)
\end{abstract}

Key words: Endothelial function, Hypertension

$\mathrm{H}$ ypertension is an independent predictor of cardiovascular disease (CVD) events. Microalbuminuria (MAU) is a marker of endothelial dysfunction and a predictor of CVD events. Endothelial dysfunction plays a key role in the pathogenesis of atherosclerosis. Carotid plaque (CP) is a marker of atherosclerosis. ${ }^{1)}$ Both MAU and CP are classified as subclinical target organ damage (OD) in hypertension. The 2013 guidelines of the European Society of Hypertension and the European Society of Cardiology (ESH/ESC) point out that any of 4 markers of OD, including MAU and CP, indicate the high-risk category of CVD. ${ }^{2)}$ It is noteworthy that the risk increases as the number of damaged organs increases. Although the relationships between MAU and endothelial dysfunction, as well as with impaired endothelial function in hypertensive patients with $\mathrm{CP}$, have been investigated in several studies, ${ }^{3,4)}$ the superposition of two OD parameters such as MAU and $\mathrm{CP}$ in association with endothelial function remains unclear.

Carotid plaque-induced endothelial dysfunction is also indicated as a predictor for CVD events. ${ }^{1)}$ A recent study has found that vascular endothelium impairment, which is a key initial step in atherogenesis, ${ }^{5)}$ is also associated with hypertension, ${ }^{6}$ and endothelial dysfunction is common in subjects with cardiovascular risk factors. ${ }^{7)}$ Endothelial function can be assessed by flow-mediated dilation (FMD). Nitroglycerin-mediated dilation (NMD) has been used as a control test for FMD when evaluating endothelial function in humans. ${ }^{8)}$ FMD is an endothelium-dependent response while NMD is an endothelium-independent response. FMD is dependent on local nitric oxide (NO) bioavailability, and NMD is dependent on exogenous NO (eg, nitroglycerin). Both are well-established methods for noninvasive assessment of vascular function. ${ }^{9}$ Recently, a few studies have demonstrated that not only FMD but also NMD is impaired in patients with atherosclerosis. ${ }^{8,10)}$ NMD may be a marker of the grade of atherosclerosis. ${ }^{8)}$ Impaired endothelium-independent vasodilation is thought to be associated with the structural vascular changes and changes in smooth muscle cells as a result of atherosclerosis. In addition, although

From the ${ }^{1}$ Department of Cardiology, Qianfoshan Hospital of Shandong Province, Shandong University, ${ }^{2}$ Shandong Academy of Medical Sciences, Jinan, and ${ }^{3}$ Department of Ultrasound, Qianfoshan Hospital of Shandong Province, Shandong University, Shandong, China.

This study was supported by the Shandong Province Outstanding Young Scientist Research Award Fund of China (BS2010YY062), the Youth Foundation of the Bureau of Public Health of Shandong Province (JW50), and Medical and Health Technology Development Program of Shandong Province (2013WSB04005).

Address for correspondence: Rui Xu, MD, Department of Cardiology, Shandong Qianfoshan Hospital, Shandong University, 16766 Jingshi Road, Jinan 250014, China. E-mail: xuruicn@hotmail.com

Received for publication August 12, 2013. Revised and accepted September 20, 2013

Released advance online J-STAGE March 14, 2014.

All rights are reserved to the International Heart Journal Association. 
impaired NMD has been demonstrated to be associated with atherosclerosis, there is little information on whether NMD of the brachial artery can serve as a marker of the progression of OD in hypertensive patients. Therefore, the aims of this study were to assess the effect of MAU on endothelial function and endothelium-independent vasodilation in hypertensive patients with $\mathrm{CP}$, and to examine the possible relationship between the clinical characteristics and endothelial function in a cohort of hypertensive patients with $\mathrm{CP}$.

\section{Methods}

Subjects and study design: Seventy-one hypertensive patients with $\mathrm{CP}$ were enrolled as the CP group in this cross-sectional study between January 2011 and January 2012, and 20 healthy people were enrolled as the normal control group (NC group). The following data were obtained: age, sex, weight, smoking status, medication information, heart rate, blood pressure, biochemical and urinary assessments, intima-media thickness (IMT), and endothelial function. Endothelial function was assessed by FMD in the brachial artery by ultrasonography, and NMD was used as a control test for FMD. The urinary albumin excretion rate (UAER), creatinine clearance rate (Ccr), and estimated glomerular filtration rate (eGFR) were calculated. The $\mathrm{CP}$ group was then divided into two groups based on their UAER: a microalbuminuria group (MAU group, $30 \mathrm{mg} / 24$ hours $<$ UAER $<300 \mathrm{mg} / 24$ hours, $n=33$ ) and a non-microalbuminuria group (NM group, UAER $<30 \mathrm{mg} / 24$ hours, $n=$ 38). We defined smokers as those who had ever smoked. Medication information was recorded as those who had taken statins or antihypertensive drugs, such as beta-blockers, calcium antagonists, and angiotensin converting enzyme inhibitors or angiotensin receptor blockers. Most of the patients were newly diagnosed with hypertension or new patients without any treatment. Those who were taking medicine such as statins or antihypertensives were suggested to discontinue the drugs for 5 half-lives before the vascular test.

The inclusion criteria were as follows: (1) patients with hypertension: systolic and diastolic blood pressure $\geq 140 / 90$ $\mathrm{mmHg}$ recorded while the patient was in a sitting position after 30 minutes of rest, or the use of antihypertensive medication, (2) those with CP: presence of plaque or maximum local carotid IMT $\geq 1.2 \mathrm{~mm},{ }^{11)}$ and (3) patients were 35 to 75 years of age. The exclusion criteria were as follows: (1) secondary hypertension, (2) massive albuminuria: UAER $\geq 300 \mathrm{mg} / 24$ hours, (3) severe heart failure or renal failure, (4) a recent history (within 6 months) of myocardial infarction or stroke or arrhythmia, and (5) those with malignant tumors. The local ethics committee approved the study, and all patients gave written informed consent for participation in the study.

Biochemical and urinary assessments: Laboratory tests, including those for measuring serum creatinine $(\mathrm{Cr})$, uric acid (UA), fasting plasma glucose (Glu), triglycerides (TG), total cholesterol (TC), high density lipoprotein (HDL), low density lipoprotein (LDL), and high-sensitivity C-reactive protein (hs$\mathrm{CRP}$ ), were performed using commercial kits employing routinely used clinical chemistry procedures. MAU was measured by rate nephelometry in the first morning urine sample with the same commercial kits and followed a standardized sample collection and testing procedure. Ccr was estimated using the
Cockcroft-Gault equation: $\mathrm{Ccr}(\mathrm{mL} / \mathrm{min})=(140$-age $) \times$ weight $\times(72 \times \mathrm{Cr})^{-1} \times(0.85$, if female $)$, and the eGFR using the MDRD (modified according to Chinese) $:^{12)}$ eGFR $\left(\mathrm{mL} / \mathrm{min} / 1.73 \mathrm{~m}^{-2}\right)=$ $186 \times \mathrm{Cr}^{-1.154} \times \operatorname{age}^{-0.203} \times(0.742$, if female $) \times(1.233$, if Chinese).

Ultrasonography: Ultrasound imaging was conducted using an Aloka alpha 10 Doppler ultrasound (Hitachi Limited, Japan) with an $11 \mathrm{MHz}$ ultrasound probe. Longitudinal ultrasonographic scans of the carotid artery included an evaluation of the right and left common carotid artery $1 \mathrm{~cm}$ proximal to the carotid bulb. In each examination, the same operator used different scanning angles to identify the greatest IMT, defined as the distance between the junction of the lumen and intima and that of the media and adventitia in the far wall of the carotid artery. Finally, 6 measurements of carotid IMT were obtained from the right and left carotid artery and were averaged to determine the mean carotid IMT. Carotid IMT was expressed in millimeters and $\mathrm{CP}$ was defined as local carotid $\mathrm{IMT} \geq 1.2 \mathrm{~mm}$.

FMD and NMD of the brachial artery are commonly used to evaluate endothelial-dependent and endothelial-independent function. The study was performed in a quiet room with the subject in a resting and supine position. The brachial artery diameter was imaged at approximately $3-5 \mathrm{~cm}$ proximal to the antecubital crease of the right arm with the forearm supinated. To create a flow stimulus in the brachial artery after baseline ultrasound imaging, a sphygmomanometer cuff was placed on the forearm and the cuff was inflated to $200 \mathrm{mmHg}$ to occlude artery flow for 4 minutes. The diameter was measured within 90 seconds after the cuff was released. All measurements were made at the end of diastole. The response to NMD was used for the determination of endothelium-independent vasodilation. All participants rested 30 minutes after the cuff was released to reach a stable baseline before data collection. After obtaining a baseline brachial artery image, a sublingual nitroglycerin tablet $(0.5 \mathrm{mg})$ was administered, and brachial artery images and measurements were repeated and obtained as detailed above. The FMD and NMD were expressed as the percentage of change in post-stimulus diameter of the artery compared to the baseline diameter $(\triangle \mathrm{FMD} \%$ and $\triangle \mathrm{NMD} \%$ ).

Statistical analysis: A statistical software package (SPSS, version 17.0, Chicago, IL, USA) was used for the analyses. All values are expressed as the mean \pm standard deviation $(\overline{\mathrm{x}} \pm \mathrm{s})$. If variables were not normally distributed, the median (quartile $_{1}$-quartile $\left.{ }_{3}\right)\left[M\left(Q_{1}-Q_{3}\right)\right]$ was expressed. The significance of differences between the groups was determined by chi-square tests for categorical variables. Univariate comparisons were performed using a $t$-test for normally distributed continuous variables. If variables were not normally distributed, the MannWhitney test was used. Data from 3 groups were compared using one-way analysis of variance (ANOVA) for normally distributed continuous variables. If variables were not normally distributed the Kruskal-Wallis test was used. Correlations were determined by Pearson's rank correlation test. If variables were not normally distributed the Spearman test was used. To identify independent determinants of $\triangle \mathrm{FMD} \%$ and $\triangle \mathrm{NMD} \%$, multiple stepwise regression analysis was performed. Statistical significance was set at $P<0.05$. 
Table I. Clinical Characteristics

\begin{tabular}{|c|c|c|c|c|}
\hline Variables & NC group & NM group & MAU group & $P$ \\
\hline $\mathrm{N}$ (male/female) & $20(12 / 8)$ & $38(24 / 14)$ & $33(14 / 19)$ & NS \\
\hline Age (years) $(\overline{\mathrm{x}} \pm \mathrm{s})$ & $59.00 \pm 6.84$ & $61.08 \pm 9.44$ & $61.70 \pm 8.48$ & NS \\
\hline Drugs $(\%)$ & -- & $14(36.84)$ & $12(36.36)$ & NS \\
\hline Statins $(\%)$ & -- & $2(5.26)$ & $2(6.06)$ & NS \\
\hline ACEIs (\%) & -- & $3(7.89)$ & $2(6.06)$ & NS \\
\hline $\operatorname{ARBs}(\%)$ & -- & $3(7.89)$ & $3(9.09)$ & NS \\
\hline$\beta$-Blockers $(\%)$ & -- & $4(10.53)$ & $3(9.09)$ & NS \\
\hline CCBs $(\%)$ & -- & $2(5.26)$ & $2(6.06)$ & NS \\
\hline Smoker $(\%)$ & $6(30.00)$ & $11(28.95)$ & $8(24.24)$ & NS \\
\hline$\Delta \mathrm{FMD} \%\left[\mathrm{M}\left(\mathrm{Q}_{1}-\mathrm{Q}_{3}\right)\right]$ & $12.78(11.38-15.78)$ & $7.69(4.38-12.94)^{*}$ & $2.56(0.00-4.55)^{* \dagger}$ & 0.000 \\
\hline$\Delta \mathrm{NMD} \%\left[\mathrm{M}\left(\mathrm{Q}_{1}-\mathrm{Q}_{3}\right)\right]$ & $21.27(21.20-22.84)$ & $13.73(9.06-24.13)$ & $10.26(6.06-15.79)^{* \pi}$ & 0.000 \\
\hline $\operatorname{IMT}(\mathrm{mm})(\overline{\mathrm{x}} \pm \mathrm{s})$ & $0.63 \pm 0.12$ & $0.75 \pm 0.13^{*}$ & $0.72 \pm 0.08^{*}$ & 0.001 \\
\hline Systolic BP $(\mathrm{mmHg})(\overline{\mathrm{x}} \pm \mathrm{s})$ & $114.05 \pm 2.63$ & $131.18 \pm 11.63^{*}$ & $135.45 \pm 16.56^{*}$ & 0.000 \\
\hline Diastolic BP (mmHg) $(\overline{\mathrm{x}} \pm \mathrm{s})$ & $72.95 \pm 2.26$ & $81.34 \pm 8.47^{*}$ & $82.24 \pm 9.25^{*}$ & 0.000 \\
\hline Creatinine $(\mu \mathrm{mol} / \mathrm{L})(\overline{\mathrm{x}} \pm \mathrm{s})$ & $79.56 \pm 11.49$ & $71.60 \pm 15.91$ & $76.02 \pm 13.26$ & NS \\
\hline Uric acid $(\mu \mathrm{mol} / \mathrm{L})(\overline{\mathrm{x}} \pm \mathrm{s})$ & $282.50 \pm 30.04$ & $326.22 \pm 78.30$ & $317.62 \pm 70.39$ & NS \\
\hline Glu $(\mathrm{mmol} / \mathrm{L})(\overline{\mathrm{x}} \pm \mathrm{s})$ & $4.98 \pm 0.43$ & $5.32 \pm 0.55$ & $6.13 \pm 1.76^{* \dagger}$ & 0.001 \\
\hline Triglyceride $(\mathrm{mmol} / \mathrm{L})\left[\mathrm{M}\left(\mathrm{Q}_{1}-\mathrm{Q}_{3}\right)\right]$ & $1.38(1.03-1.68)$ & $1.47(0.92-2.10)$ & $1.33(1.03-1.64)$ & NS \\
\hline Total cholesterol $(\mathrm{mmol} / \mathrm{L})(\overline{\mathrm{x}} \pm \mathrm{s})$ & $4.54 \pm 0.36$ & $5.00 \pm 0.87$ & $5.31 \pm 0.91^{*}$ & 0.005 \\
\hline $\mathrm{HDL}(\mathrm{mmol} / \mathrm{L})(\overline{\mathrm{x}} \pm \mathrm{s})$ & $1.38 \pm 0.20$ & $1.32 \pm 0.31$ & $1.36 \pm 0.39$ & NS \\
\hline $\mathrm{LDL}(\mathrm{mmol} / \mathrm{L})(\overline{\mathrm{x}} \pm \mathrm{s})$ & $2.58 \pm 0.30$ & $3.09 \pm 0.66^{\S}$ & $3.11 \pm 0.77^{\S}$ & 0.009 \\
\hline hs-CRP $(\mathrm{mmol} / \mathrm{L})\left[\mathrm{M}\left(\mathrm{Q}_{1}-\mathrm{Q}_{3}\right)\right]$ & $0.56(0.40-0.78)$ & $1.06(0.59-1.75)^{*}$ & $0.72(0.45-1.89)$ & 0.046 \\
\hline $\log \operatorname{UAER}(\overline{\mathrm{x}} \pm \mathrm{s})$ & $1.23 \pm 0.98$ & $1.35 \pm 0.08$ & $1.88 \pm 0.31^{* \dagger}$ & 0.000 \\
\hline $\operatorname{Ccr}(\mathrm{mL} / \mathrm{min})(\overline{\mathrm{x}} \pm \mathrm{s})$ & $91.15 \pm 18.86$ & $93.61 \pm 24.12$ & $93.62 \pm 27.18$ & NS \\
\hline eGFR $\left(m L \min ^{-1} \operatorname{per} 1.73 \mathrm{~m}^{2}\right)(\overline{\mathrm{x}} \pm \mathrm{s})$ & $104.10 \pm 23.83$ & $119.23 \pm 27.35$ & $119.76 \pm 24.78$ & NS \\
\hline
\end{tabular}

ACEIs indicates angiotensin converting enzyme inhibitors; ARBs, angiotensin receptor blockers; CCB, calcium channel blockers; FMD, flow-mediated dilation; NMD, nitroglycerin-mediated dilation; IMT, intima-media thickness; BP, blood pressure; Glu, fasting plasma glucose; HDL, high density lipoprotein; LDL, low density lipoprotein; hs-CRP, high-sensitivity C-reactive protein; UAER, urinary albumin excretion rates; Ccr, creatinine clearance rate; and eGFR, estimated glomerular filtration rate. Compared with NC group, ${ }^{*} P<0.01,{ }^{\S} P<0.05$; Compared with NM group, ${ }^{\dagger} P<0.01,{ }^{\mathbb{I}} P<0.05$.

\section{Results}

Comparison of clinical characteristics of patients in the MAU, NM, and NC groups: The MAU group, NM group, and NC group consisted of 33,38 , and 20 patients, respectively. Because the distribution of UAER was not normal, it was logtransformed before statistical analysis. Table I shows the clinical characteristics of all patients. Sex, age, medication information, smoking status, Cr levels, UA levels, TG levels, HDL levels, Ccr, and eGFR did not differ significantly among the 3 groups.

IMT, SBP, DBP, and LDL levels were significantly increased in both the NM group and MAU group $(P<0.01)$. Glu levels, TC levels and log UAER were significantly increased only in the MAU group $(P<0.01)$. On the whole, the $\triangle$ FMD $\%$ of the CP group was obviously lower than that of the $\mathrm{NC}$ group [4.55 (2.35-8.42)\% versus 12.78 (11.38-15.78)\%, $P<$ $0.01]$. The $\triangle \mathrm{NMD} \%$ of the $\mathrm{CP}$ group exhibited a trend similar to that for $\triangle$ FMD\% [11.54 (7.32-19.43)\% versus 21.27 (21.20$22.84) \%, P<0.01]$. Compared to the NC group, the $\Delta$ FMD $\%$ was significantly reduced in the NM group [12.78 (11.38$15.78) \%$ versus $7.69(4.38-12.94) \%, P<0.001]$, and there was also a significant difference between the NM group and MAU group [7.69 (4.38-12.94)\% versus $2.56(0.00-4.55) \%, P<$ $0.001]$. Compared to the NM group, the $\triangle \mathrm{NMD} \%$ was significantly reduced in the MAU group [13.73 (9.06-24.13)\% versus 10.26 (6.06-15.79)\%, $P=0.026]$. However, no significant difference was observed between the NC group and NM group [21.27 (21.20-22.84)\% versus $13.73(9.06-24.13) \%, \mathrm{NS}]$.

Relationship between biochemical parameters and FMD: As listed in Table II, we observed that $\triangle \mathrm{FMD} \%$ demonstrated sig-
Table II. Relationship Between Biochemical Parameters and Endothelial Function

\begin{tabular}{lcccc}
\hline \multirow{2}{*}{ Variables } & \multicolumn{2}{c}{$\Delta$ FMD $\%$} & \multicolumn{2}{c}{$\Delta$ NMD $\%$} \\
\hline Age & -0.085 & NS & -0.207 & 0.049 \\
IMT & -0.217 & 0.040 & -0.296 & 0.004 \\
Systolic BP & -0.442 & 0.000 & -0.271 & 0.009 \\
Diastolic BP & -0.186 & NS & -0.112 & NS \\
Creatinine & 0.186 & NS & 0.049 & NS \\
Uric acid & -0.174 & NS & -0.013 & NS \\
Glu & -0.356 & 0.001 & -0.220 & 0.036 \\
Triglycerides & 0.110 & NS & 0.075 & NS \\
Total cholesterol & -0.381 & 0.000 & -0.182 & NS \\
HDL & 0.073 & NS & 0.065 & NS \\
LDL & -0.267 & 0.011 & -0.113 & NS \\
hs-CRP & -0.234 & 0.026 & -0.100 & NS \\
log UAER & -0.596 & 0.000 & -0.311 & 0.003 \\
Ccr & 0.030 & NS & 0.054 & NS \\
eGFR & -0.076 & NS & -0.020 & NS \\
\hline
\end{tabular}

FMD indicates flow-mediated dilation; NMD, nitroglycerin-mediated dilation; IMT, intima-media thickness; BP, blood pressure; Glu, fasting plasma glucose; HDL, high density lipoprotein; LDL, low density lipoprotein; hs-CRP, high-sensitivity C-reactive protein; UAER, urinary albumin excretion rates; Ccr, creatinine clearance rate; and eGFR, estimated glomerular filtration rate.

nificant correlations with $\operatorname{SBP}(r=-0.442, P<0.001)$, IMT $(r$ $=-0.217, P=0.040)$, Glu $(r=-0.356, P=0.001)$, TC $(r=$ $-0.381, P<0.001)$, LDL $(r=-0.267, P=0.011)$, hs-CRP $(r=$ $-0.234, P=0.026)$ and $\log \operatorname{UAER}(r=-0.596, P<0.001)$, respectively, in all subjects in this study. Representative scattergrams are shown in Figure 1. 

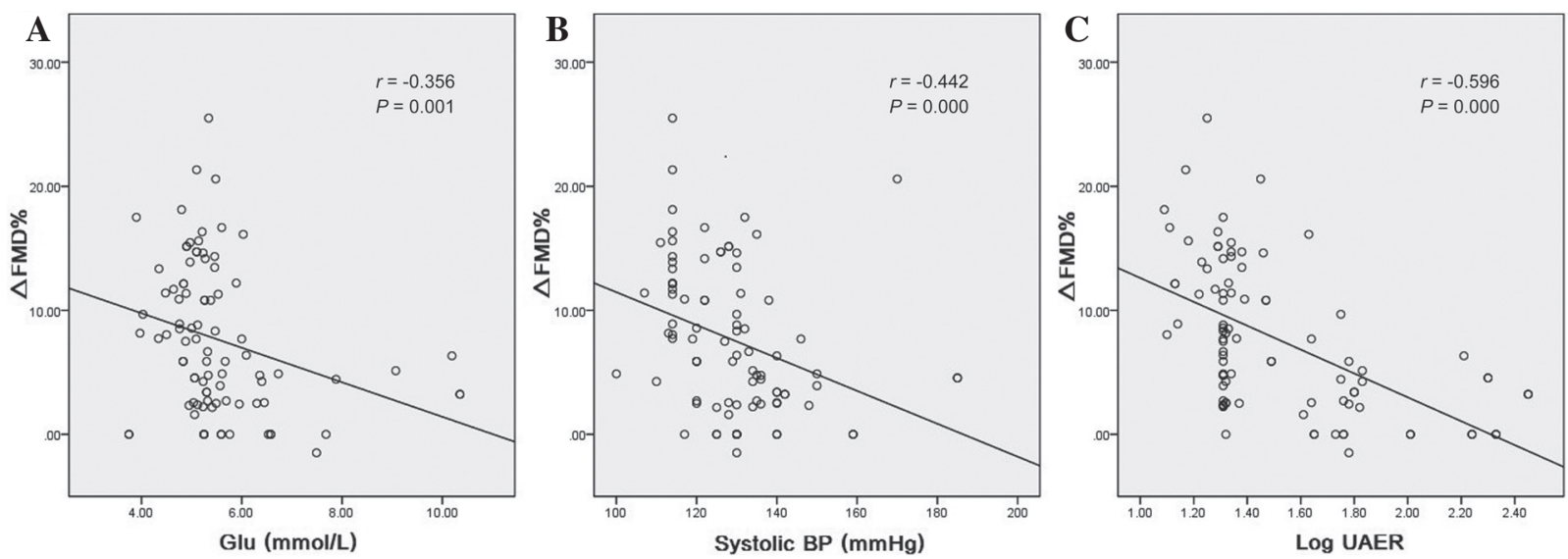

Figure 1. A: $\triangle \mathrm{FMD} \%$ demonstrated significant correlations with Glu. B: $\triangle \mathrm{FMD} \%$ demonstrated significant correlations with Systolic BP. C: $\triangle \mathrm{FMD} \%$ demonstrated significant correlations with Log UAER. FMD indicates flow-mediated dilation; BP, blood pressure; Glu, fasting plasma glucose; and UAER, urinary albumin excretion rates.
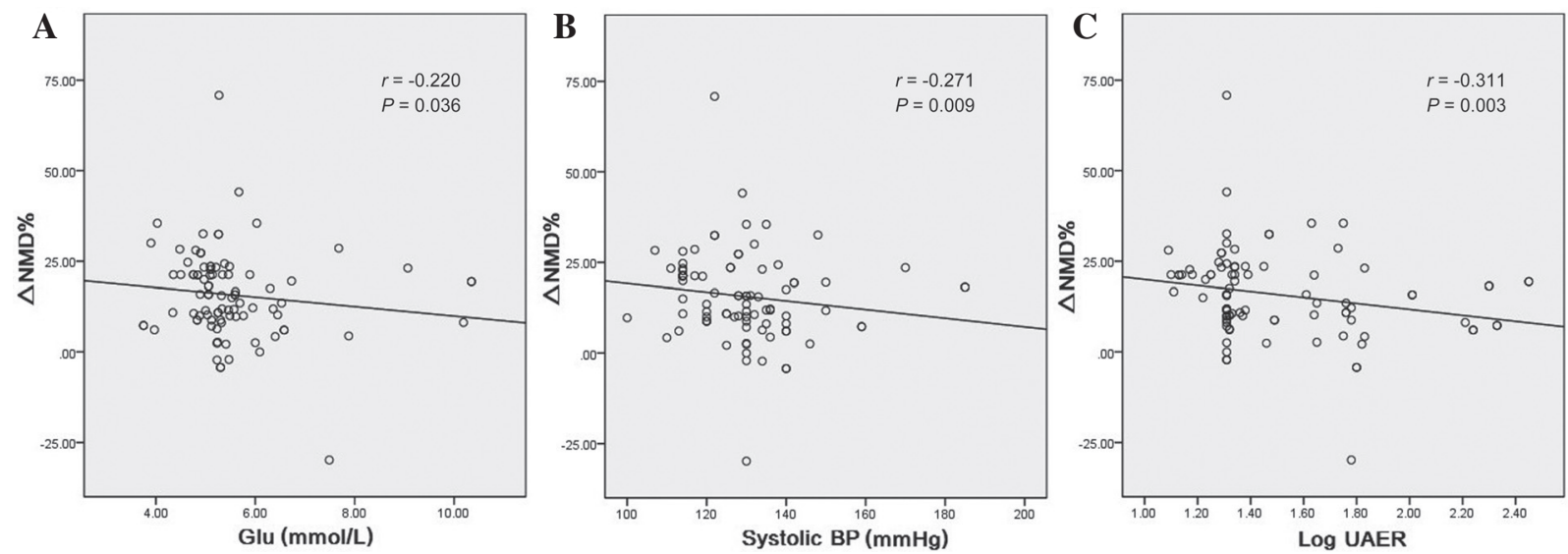

Figure 2. A: $\triangle \mathrm{NMD} \%$ demonstrated significant correlations with Glu. B: $\triangle \mathrm{NMD} \%$ demonstrated significant correlations with Systolic BP. C: $\triangle \mathrm{NMD} \%$ demonstrated significant correlations with the Log UAER. NMD indicates nitroglycerin-mediated dilation; Glu, fasting plasma glucose; BP, blood pressure; and UAER, urinary albumin excretion rates.

Stepwise multiple linear regression analysis was performed to investigate whether the biochemical parameters are associated with FMD independently. Multiple regression analysis showed that UAER and TC were independent determinants of FMD (adjusted $R^{2}=0.329, P=0.027$ ). The results are presented in Table III.

Relationship between biochemical parameters and NMD: As listed in Table II, the $\triangle \mathrm{NMD} \%$ demonstrated significant correlations with age $(r=-0.207, P=0.049), \operatorname{SBP}(r=-0.271, P=$ $0.009)$, IMT $(r=-0.296, P=0.004)$, Glu $(r=-0.220, P=$ $0.036)$, and $\log$ UAER $(r=-0.311, P=0.003)$. Representative scattergrams are shown in Figure 2.

Stepwise multiple linear regression analysis was performed to identify factors associated with NMD and biochemical parameters. Multiple regression analysis showed that the UAER and age were independent determinants of NMD (adjusted $\left.R^{2}=0.076, P=0.044\right)$. The results are listed in Table III. Relationship between biochemical parameters and microalbuminuria: Table IV shows that UAER was associated with $\operatorname{SBP}(r=0.546, P<0.001)$, DBP $(r=0.284, P=0.006)$, Glu $(r$ $=0.466, P<0.001)$, TC $(r=0.288, P=0.006)$, and LDL $(r=$
$0.269, P=0.010)$. Representative scattergrams are shown in Figure 3.

In multivariate regression analysis, $\triangle \mathrm{FMD} \%$, SBP, hsCRP, Glu, CR, DBP, IMT, TG, and weight were independently associated with UAER (adjusted $R^{2}=0.688, P=0.035$ ) (Table III).

\section{Discussion}

Patients with hypertension and CP in our study showed a higher prevalence of MAU (46.5\%) compared with Sun's study. ${ }^{13)}$ In their study, the proportion of patients with MAU was $18.6 \%$ in the hypertension population. This can perhaps be explained in terms of endothelial dysfunction, which always appears in patients with $\mathrm{CP}$, and microalbumin can leak from the damaged vascular endothelium. This prompted us to surmise that hypertensive patients with $\mathrm{CP}$ are a high-risk group for early renal damage.

To our knowledge, there are presently two methods to evaluate endothelial function. One is the indirect method of 
Table III. Multivariate Regression Analysis Results

\begin{tabular}{ccccc}
\hline $\begin{array}{c}\text { Dependent } \\
\text { variable }\end{array}$ & $\begin{array}{c}\text { Independent } \\
\text { variable }\end{array}$ & $\begin{array}{c}\text { Standardized } \\
\text { coefficients }\end{array}$ & $t$ & $P$ \\
\hline \multirow{2}{*}{$\Delta$ FMD\% } & constant & 27.913 & 8.080 & $0.000^{*}$ \\
& LogUAER & -0.496 & -5.480 & $0.000^{*}$ \\
& Total cholesterol & -0.203 & -2.243 & $0.027^{\S}$ \\
conMD\% & constant & 45.235 & 4.501 & $0.000^{*}$ \\
& LogUAER & -0.212 & -2.084 & $0.040^{\S}$ \\
& Age & -0.208 & -2.044 & $0.044^{\S}$ \\
& constant & 1.443 & 4.594 & $0.000^{*}$ \\
& SFMD\% & -0.246 & -3.640 & $0.000^{*}$ \\
& Systolic BP & 0.665 & 6.654 & $0.000^{*}$ \\
& hs-CRP & 0.331 & 4.875 & $0.000^{*}$ \\
& Glu & 0.183 & 2.704 & $0.008^{*}$ \\
& Creatinine & -0.210 & -3.303 & $0.001^{*}$ \\
& Diastolic BP & -0.308 & -3.260 & $0.002^{*}$ \\
& IMT & -0.212 & -3.189 & $0.002^{*}$ \\
& Triglycerides & -0.146 & -2.395 & $0.019^{\S}$ \\
& Weight & -0.137 & -2.144 & $0.035^{\S}$ \\
\hline
\end{tabular}

FMD indicates flow-mediated dilation; NMD, nitroglycerin-mediated dilation; UAER, urinary albumin excretion rates; BP, blood pressure; hs$\mathrm{CRP}$, high-sensitivity $\mathrm{C}$-reactive protein; Glu, fasting plasma glucose; and IMT, intima-media thickness. ${ }^{*} P<0.01,{ }^{\S} P<0.05$.
Table IV. Relationship Between Biochemical Parameters and Microalbuminuria

\begin{tabular}{ccc}
\hline Variables & \multicolumn{2}{c}{ Log UAER } \\
& $r$ & $P$ \\
\hline Age & 0.089 & NS \\
IMT & 0.117 & NS \\
Systolic BP & 0.546 & 0.000 \\
Diastolic BP & 0.284 & 0.006 \\
Creatinine & 0.124 & NS \\
Uric acid & 0.072 & NS \\
Glu & 0.466 & 0.000 \\
Triglycerides & -0.243 & NS \\
Total cholesterol & 0.304 & 0.003 \\
HDL & -0.182 & NS \\
LDL & 0.227 & 0.030 \\
hs-CRP & 0.149 & NS \\
Ccr & -0.011 & NS \\
eGFR & 0.104 & NS \\
\hline
\end{tabular}

IMT indicates intima-media thickness; BP, blood pressure; Glu, fasting plasma glucose; HDL, high density lipoprotein; LDL, low density lipoprotein; hs-CRP, high-sensitivity C-reactive protein; UAER, urinary albumin excretion rates; Ccr, creatinine clearance rate; and eGFR, estimated glomerular filtration rate.
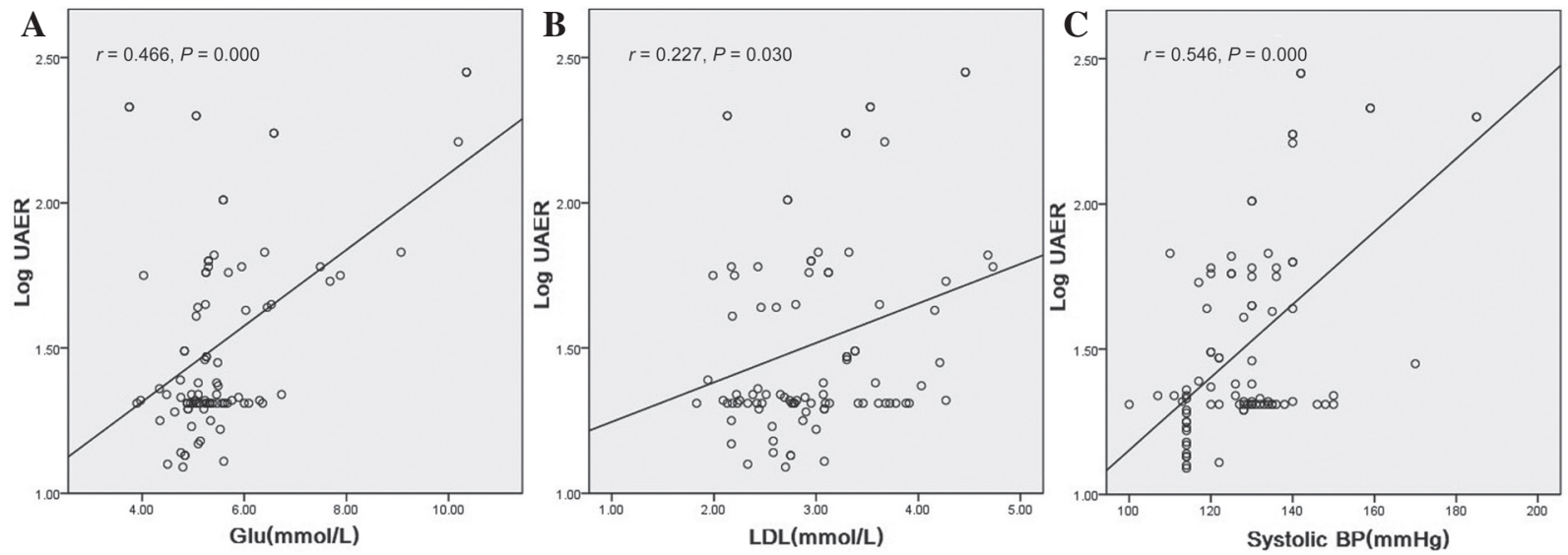

Figure 3. A: Log UAER demonstrated significant correlations with Glu. B: Log UAER demonstrated significant correlations with the LDL. C: Log UAER demonstrated significant correlations with the Systolic BP. Glu indicates fasting plasma glucose; LDL, low density lipoprotein; BP, blood pressure; and UAER, urinary albumin excretion rates.

laboratory examination which includes serum NO and endothelin-1 (ET-1). The other one is FMD, which is a direct method involving ultrasonography. Because exogenous vasodilator substances (eg, NO) act on vascular smooth muscle cells as well as endogenous NO, NMD is usually used as a control test for FMD. ${ }^{8)}$ Ultrasonography was used in this study because of its noninvasive and more direct result. FMD, which can be expressed as the rate of change of vessel diameter before and after arm tying, is a kind of vasodilation that depends on the integrity of the structure and function of normal endothelium. While NMD is a kind of vasodilation that depends on exogenous drugs such as NO which can relax the vascular smooth muscle directly, it can be expressed as the rate of change of vessel diameter before and after taking nitroglycerin.

The main finding of this study is that the FMD was significantly decreased in hypertensive patients with $\mathrm{CP}$, especially in those also with MAU. However, the NMD was not significantly changed in hypertensive patients with $\mathrm{CP}$ only, but decreased in patients with MAU. At present, most studies have described an association between lower FMD and hypertension/atherosclerosis. However, there is still some difference of opinion about NMD among several studies. In Veerabhadrappa's ${ }^{14)}$ study, masked-hypertensive patients had a diminished FMD compared to the true prehypertensive patients, but did not have a diminished NMD. However, another study ${ }^{15)}$ involving 800 subjects demonstrated that both FMD and NMD were impaired in these patients although they had no symptoms but did have risk factors for atherosclerosis. Similarly, Gokce ${ }^{16)}$ found that hypertensive patients had endothelial dysfunction which included diminished FMD and NMD. A recent study showed that NMD was associated with cardiovascular risk factors and CVD and might be a marker of the grade of atherosclerosis. ${ }^{8)}$ Therefore, it is possible that the process of the decreases in FMD and NMD is inconsistent in different stages of hypertension and grades of atherosclerosis. Although highnormal blood pressure can injure the endothelium due to in- 
creased pressure on the vessel wall, secretory function is normal and both FMD and NMD are at regular levels. With the progression of hypertension or emergence of subclinical target organ damage, the endothelial secretory dysfunction caused reflex vasodilator dysfunction. However, the vascular smooth muscle cells were not affected, and the addition of exogenous NO can dilate the vascular wall. Thus, the FMD was decreased but the NMD was normal. Furthermore, when there are more subclinical target organ damage emerged, such as CP together with MAU, NMD maybe impaired as FMD. To explain this, some mechanisms have been postulated such as the impairment of vascular smooth muscle cell function, or the soluble guanylate cyclase/cyclic guanosine monophosphate signaling system. ${ }^{17)}$ Overall, it is suggested that impairment of the arterial wall is not limited to the endothelium layer only. The vascular smooth muscle may also be damaged simultaneously.

Secondly, multiple regression analysis showed that the UAER and TC were risk factors of FMD and that the UAER and age were risk factors of NMD. It is known the permeability of the capillary basement membrane is increased when the endothelium experiences a minor injury. Albumin can leak from the basement membrane causing the appearance of MAU. At the same time, the increased activity of protein kinase $\mathrm{C}^{18)}$ could inhibit NO synthetase, and thus the shortage of NO aggravated the endothelial dysfunction. This could be one of the reasons why UAER was an independent risk factor for endothelial dysfunction in this study. A previous study ${ }^{19)}$ reported that statins can improve endothelial function by reducing TC. We have found that TC was a risk factor of FMD but not NMD. This result is similar to the findings of Liu, et $a l^{20)}$ who found that the reduction of TC and weight loss caused by drugs can improve FMD but does not affect NMD. This can perhaps be explained as the diminished FMD is caused by the impaired endothelium itself while the NMD can be regulated by exogenous vasodilator substances (eg, NO) through the vascular smooth muscle. Therefore, reduced endothelial function caused by elevated serum lipid levels affects FMD more severely than NMD. Furthermore, age is also a risk factor of NMD. With increasing age, the diastolic function of the vascular smooth muscle gradually decreases due to the exacerbation of atherosclerosis, which independently affects the function of NMD.

The relationships between biochemical parameters and FMD or NMD indicate that UAER, TC, and age are common risk factors of FMD and NMD. We have also found that the $\triangle$ FMD \% demonstrated significant correlations with SBP, IMT, Glu, LDL, and hs-CRP, and that the $\triangle$ NMD\% demonstrated significant correlations with the SBP, IMT, and Glu. These results are similar to those of Maruhashi, et al. ${ }^{8)}$ As mentioned above, endothelial dysfunction is a key initial step in atherogenesis. ${ }^{5)}$ Although NMD is not a consequence of endothelial dysfunction, it is a kind of vascular smooth muscle dysfunction. It has been shown that NMD was impaired in adults with cardiovascular risk factors. ${ }^{10)}$ Like previous observations, our results showed that cardiovascular risk factors, such as SBP, IMT, Glu, LDL, and hs-CRP are significantly correlated with FMD and NMD. These findings suggest that endothelial dysfunction can help identify patients at high-risk for atherogenesis, and this may explain the pathogenesis of endothelial dysfunction.

Thirdly, the study examined the risk factors of MAU in hypertensive patients with $\mathrm{CP}$. The results confirmed that $\triangle$ FMD $\%$, SBP, hs-CRP, Glu, CR, DBP, IMT, TG, and weight were independent risk factors for MAU, as previously reported. ${ }^{21)}$ MAU aggravates endothelial dysfunction and thus more microalbumin can leak from the impaired endothelium. Endothelial dysfunction leads to the MAU. $\triangle$ FMD $\%$ and the incrassate IMT, which can be regarded as the consequences of endothelial dysfunction, are important risk factors of MAU. In addition, a high BP is associated with MAU. Elevated BP could cause peritubular capillary loss, which leads to interstitial fibrosis, and peritubular capillary loss is reported to be correlated with impairment of kidney function. ${ }^{22)}$ Therefore, abnormalities in small vessels might affect MAU. Fortunately, a randomized trial showed that early detection of MAU and blood pressure control can slow the progression of CVD. ${ }^{23)} \mathrm{Di}-$ abetic patients are at high risk of developing complications such as renal damage or diabetic nephropathy. High serum Glu can lead to inflammation and increased oxidative stress. Both high serum Glu and inflammatory factors, especially hs-CPR, may contribute to the pathogenesis of diabetic complications including nephropathy. At the early stage of nephropathy, the appearance of MAU means that injury to the endothelium has been started. In order to estimate the global risk burden of patients with high blood pressure, it is necessary to detect and measure Glu and glycosylated hemoglobin, especially in patients with high levels of MAU. In addition, dyslipidemia affects the process of renal damage because of the lipid deposition in the glomerular basement membrane, which can stimulate cell proliferation and extracellular matrix formation. This could lead to glomerulosclerosis due to inflammatory cell infiltration and foam cell formation. Dyslipidemia is often accompanied by being overweight and insulin resistance, both of which can cause endothelial dysfunction and were found to be correlated with MAU. ${ }^{24)}$

Finally, there are two other points that should be mentioned. One is that both eGFR and Ccr were normal when MAU appeared. This suggests that MAU was an earlier marker of early renal damage. The other is that the IMT in the MAU group and NM group was not significantly different. It would be preferable to assess patients comprehensively according to each subclinical target organ damage rather than to IMT only.

Our study has several limitations. Although we observed differences in endothelial function in hypertensive patients with CP and MAU, the sample size was small and the subgroup analysis was not comprehensive. The results would perhaps be more robust by expanding the sample size and enrolling patients with other subclinical target organ damage. On the other hand, in addition to conventional antihypertensive treatment, follow-up after drug treatment that is directed towards risk factors is needed. Greater clinical value will be achieved if the changes in endothelial function are detected before and after treatment. In addition, due to the study design, to detect the role of MAU in arterial endothelial dysfunction in hypertensive patients with $\mathrm{CP}$, we did not enroll hypertensive patients without CP. However, some studies ${ }^{3,25,26)}$ have shown that endothelial function was decreased in patients with $\mathrm{CP}$ compared with those without $\mathrm{CP}$ and that FMD was independently associated with $\mathrm{CP}$. We may study hypertensive patients without $\mathrm{CP}$ to investigate the relationship between NMD and subclinical target $\mathrm{OD}$ in hypertensive patients in the future.

In conclusion, we studied whether the endothelium-de- 
pendent vasodilation or the endothelium-independent vasodilation is damaged in hypertensive patients with CP and MAU. However, in patients with CP only, endothelium-independent vasodilation was normal. Therefore, in addition to other standard diagnostic procedures, endothelial function, MAU and CP should be measured in hypertensive patients in order to estimate the global risk factor burden rather than focus entirely on the severity of blood pressure elevation. It is important to identify high-risk patients and to improve the prognosis of patients with early treatment. Endothelial function in hypertensive patients with $\mathrm{CP}$ is decreased, and MAU may aggravate the arterial dysfunction.

\section{REFERENCES}

1. Polak JF, Szklo M, Kronmal RA, et al. The value of carotid artery plaque and intima-media thickness for incident cardiovascular disease: the multi-ethnic study of atherosclerosis. J Am Heart Assoc 2013; 2: e000087.

2. Mancia G, Fagard R, Narkiewicz K, et al. 2013 ESH/ESC guidelines for the management of arterial hypertension: the Task Force for the Management of Arterial Hypertension of the European Society of Hypertension (ESH) and of the European Society of Cardiology (ESC). Eur Heart J 2013; 34: 2159-219.

3. Rundek T, Hundle R, Ratchford E, et al. Endothelial dysfunction is associated with carotid plaque: a cross-sectional study from the population based Northern Manhattan Study. BMC Cardiovasc Disord 2006; 6: 35.

4. Yokoyama H, Sone H, Saito K, Yamada D, Honjo J, Haneda M. Flow-mediated dilation is associated with microalbuminuria independent of cardiovascular risk factors in type 2 diabetes - interrelations with arterial thickness and stiffness. J Atheroscler Thromb 2011; 18: 744-52.

5. Ross R. Atherosclerosis -- an inflammatory disease. N Engl J Med 1999; 340: 115-26. (Review)

6. Drexler H, Hornig B. Endothelial dysfunction in human disease. J Mol Cell Cardiol 1999; 31: 51-60. (Review)

7. Celermajer DS, Sorensen KE, Gooch VM, et al. Non-invasive detection of endothelial dysfunction in children and adults at risk of atherosclerosis. Lancet 1992; 340: 1111-5.

8. Maruhashi T, Soga J, Fujimura N, et al. Nitroglycerine-induced vasodilation for assessment of vascular function: a comparison with flow-mediated vasodilation. Arterioscler Thromb Vasc Biol 2013; 33: 1401-8.

9. Corretti MC, Anderson TJ, Benjamin EJ, et al. Guidelines for the ultrasound assessment of endothelial-dependent flow-mediated vasodilation of the brachial artery: a report of the International Brachial Artery Reactivity Task Force. J Am Coll Cardiol 2002; 39: 257-65.

10. Raitakari OT, Seale JP, Celermajer DS. Impaired vascular responses to nitroglycerin in subjects with coronary atherosclerosis. Am J Cardiol 2001; 87: 217-9.
11. Zhang M, Zhang Y, Gao YH, Zhang YY, Ge ZM, Ji XP. Remodeling of carotid atherosclerosis by high resolution ultrasonography. Chin J Ultrasonogr 2002; 11: 229-31. (Chinese)

12. Chinese eGFR Investigation Collaboration. Modification and evaluation of MDRD estimating equation for Chinese patients with chronic kidney disease. Chin J Nephrol 2006; 22: 589-95.

13. Sun NL, Wang HY, Zhu DL, Liao YH, Lin SG, Chen XP. Association between albuminuria and blood pressure level in patients with essential hypertension. Chin J Nephrol 2010; 26: 762-5.

14. Veerabhadrappa P, Diaz KM, Feairheller DL, et al. Endothelialdependent flow-mediated dilation in African Americans with masked-hypertension. Am J Hypertens 2011; 24: 1102-7.

15. Adams MR, Robinson J, McCredie R, et al. Smooth muscle dysfunction occurs independently of impaired endothelium-dependent dilation in adults at risk of atherosclerosis. J Am Coll Cardiol 1998; 32: 123-7.

16. Gokce N, Holbrook M, Duffy SJ, et al. Effects of race and hypertension on flow-mediated and nitroglycerin-mediated dilation of the brachial artery. Hypertension 2001; 38: 1349-54.

17. Patel PD, Velazquez JL, Arora RR. Endothelial dysfunction in African-Americans. Int J Cardiol 2009; 132: 157-72. (Review)

18. Mehta NN, Sheetz M, Price K, et al. Selective PKC beta inhibition with ruboxistaurin and endothelial function in type-2 diabetes mellitus. Cardiovasc Drugs Ther 2009; 23: 17-24.

19. Pretnar-Oblak J, Sebestjen M, Sabovic M. Statin treatment improves cerebral more than systemic endothelial dysfunction in patients with arterial hypertension. Am J Hypertens 2008; 21: 674-8.

20. Liu J, Sun N, Yang S, Ma Z, Yang J. Effect of orlistat-assisted weight loss on endothelium-dependent vasodilation in obese Chinese subjects with hypertension. Clin Exp Hypertens 2010; 32: 395-9.

21. Ukena C, Mahfoud F, Kindermann M, et al. Smoking is associated with a high prevalence of microalbuminuria in hypertensive highrisk patients: data from I-SEARCH. Clin Res Cardiol 2010; 99: 825-32.

22. Metcalfe W. How does early chronic kidney disease progress? A background paper prepared for the UK Consensus Conference on early chronic kidney disease. Nephrol Dial Transplant 2007; 22: ix26-30. (Review)

23. Ibsen $\mathrm{H}$, Olsen $\mathrm{MH}$, Wachtell $\mathrm{K}$, et al. Reduction in albuminuria translates to reduction in cardiovascular events in hypertensive patients: losartan intervention for endpoint reduction in hypertension study. Hypertension 2005; 45: 198-202.

24. Lteif AA, Han K, Mather KJ. Obesity, insulin resistance, and the metabolic syndrome: determinants of endothelial dysfunction in whites and blacks. Circulation 2005; 112: 32-8.

25. Irace C, Carallo C, Loprete A, Tripolino C, Scavelli F, Gnasso A. Delayed flow-mediated vasodilation and carotid atherosclerosis. Eur J Clin Invest 2013; 43: 49-55.

26. Nakamura T, Kitta Y, Uematsu M, et al. Ultrasound assessment of brachial endothelial vasomotor function in addition to carotid plaque echolucency for predicting cardiovascular events in patients with coronary artery disease. Int J Cardiol 2013; 167: 55560. 